\title{
A cohesive law for interfaces in graphene/hexagonal boron nitride heterostructure
}

\author{
Chenxi Zhang, ${ }^{1}$ Jun Lou, ${ }^{2}$ and Jizhou Song ${ }^{3, a)}$ \\ ${ }^{1}$ Department of Mechanical and Aerospace Engineering, University of Miami, Coral Gables, \\ Florida 33146, USA \\ ${ }^{2}$ Department of Materials Science and NanoEngineering, Rice University, Houston, Texas 77251, USA \\ ${ }^{3}$ Department of Engineering Mechanics and Soft Matter Research Center, Zhejiang University, \\ Hangzhou 310027, China
}

(Received 18 December 2013; accepted 28 March 2014; published online 10 April 2014)

\begin{abstract}
Graphene/hexagonal boron nitride (h-BN) heterostructure has showed great potential to improve the performance of graphene device. We have established the cohesive law for interfaces between graphene and monolayer or multi-layer h-BN based on the van der Waals force. The cohesive energy and cohesive strength are given in terms of area density of atoms on corresponding layers, number of layers, and parameters in the van der Waals force. It is found that the cohesive law in the graphene/multi-layer h-BN is dominated by the three h-BN layers which are closest to the graphene. The approximate solution is also obtained to simplify the expression of cohesive law. These results are very useful to study the deformation of graphene/h-BN heterostructure, which may have significant impacts on the performance and reliability of the graphene devices especially in the areas of emerging applications such as stretchable electronics. (C) 2014 AIP Publishing LLC. [http://dx.doi.org/10.1063/1.4870825]
\end{abstract}

\section{INTRODUCTION}

Since its fabrication, identification, and characterization in 2004, ${ }^{1}$ graphene has been stimulating scientists' and engineers' imagination and passion for nanoscience and nanotechnology. Owing to its fascinating electrical, thermal, and mechanical properties, graphene has shown great promise for a multitude of applications, ranging from flexible and invisible displays, nanoelectronic components, nanosensors, to energy conversion and storage devices. Although the feasibility of constructing free-standing graphene sheet has been demonstrated, ${ }^{2-4}$ most applications will either require graphene to be grown on ${ }^{5-7}$ or transferred ${ }^{8-10}$ to a supporting substrate. However, the presence of substrates leads to a significant reduction in electron mobility ${ }^{1,11,12}$ from $\sim 100000 \mathrm{~cm}^{2} \mathrm{~V}^{-1} \mathrm{~s}^{-1}$ to $\sim 1000 \mathrm{~cm}^{2} \mathrm{~V}^{-1} \mathrm{~s}^{-1}$ due to the charged surface states and impurities, ${ }^{13-16}$ surface roughness, ${ }^{17,18}$ and surface optical phonons. ${ }^{19,20}$ One solution to allow a substrate-supported geometry while retaining the quality achieved with a suspended sample is to use graphene/hexagonal boron nitride (h-BN) heterostructure, where h-BN serves as a dielectric layer between graphene and substrate to reduce the substrate influences on graphene. The h-BN's strong in-plane bonds, large band gap, and planar structure provide an ideal flat, insulating, and inert surface, isolating the graphene from the substrate. ${ }^{21-23}$ It is shown that graphene/h-BN heterostructure exhibits electron mobility as high as $\sim 60000 \mathrm{~cm}^{2} \mathrm{~V}^{-1} \mathrm{~s}^{-1}$, an order of magnitude higher than the commonly reported substrate-supported graphene. ${ }^{24}$

The use of graphene/h-BN heterostructure for graphene electronics has attracted much interest from science and

\footnotetext{
a) Author to whom correspondence should be addressed. Electronic mail: jzsong@gmail.com
}

engineering communities. Most of the existing studies on graphene/h-BN heterostructure focus on the electrical, ${ }^{24-31}$ magnetic, ${ }^{32,33}$ and thermal properties, ${ }^{34}$ as well as its behaviors when applied in field effect transistor. ${ }^{35,36}$ In order to utilize graphene/h-BN heterostructure in graphene electronics, mechanical properties of this heterostructure are critical because it is to be used as structural and functional components in electronics. Moreover, mechanical properties (or deformations) of graphene/h-BN are strongly tied to its electrical performance. Therefore, understanding the mechanical properties of graphene/h-BN, where the interface plays a key role, is crucial in enabling future high-quality graphene applications such as stretchable electronics.

Although interfacial sliding and/or buckling of graphene on polymer substrates have been observed experimentally, ${ }^{37-39}$ the studies on mechanical modeling for interfaces in graphene/h-BN heterostructure are rarely seen. The objective of this paper is to study graphene/h-BN heterostructure to understand the interaction between graphene and h-BN. The cohesive law for the interface between graphene and monolayer h-BN is established in Sec. II. Section III develops the cohesive law for the interface between graphene and multilayer h-BN.

\section{COHESIVE LAW FOR GRAPHENE/MONOLAYER h-BN}

Following the approaches of Jiang et al. and Lu et al. for single-wall carbon nanotubes (CNTs) and polymer matrix, ${ }^{40-42}$ we model both graphene and monolayer h-BN as homogenized solids and infinitely large in the in-plane directions. The interlayer bonding between graphene and $\mathrm{h}-\mathrm{BN}$ is due to van der Waals interaction, ${ }^{43}$ which is usually represented by the Lennard-Jones 6-12 potential 


$$
V(r)=4 \varepsilon\left(\frac{\sigma^{12}}{r^{12}}-\frac{\sigma^{6}}{r^{6}}\right),
$$

where $V(r)$ is the energy between a pair of atoms of distance $r, \sqrt[6]{2} \sigma$ is the equilibrium distance between two atoms and $\varepsilon$ is the bond energy at the equilibrium distance. For a pair of carbon and nitrogen atoms, the bond energy and equilibrium distance are $\varepsilon_{C-N}=0.004068 \mathrm{eV}$ and $\sigma_{C-N}=0.3367 \mathrm{~nm}$, respectively. ${ }^{44}$ For a pair of carbon and boron atoms, they are $\varepsilon_{C-B}=0.003294 \mathrm{eV}$ and $\sigma_{C-B}=0.3411 \mathrm{~nm}^{44}$

To describe van der Waals interactions between graphene and monolayer h-BN, both graphene and monolayer h-BN are modeled as a homogenous layer with $\rho_{C}=\frac{4}{3 \sqrt{3} l_{C}^{2}}$ denoting the number of carbon atoms per unit area on graphene and $\rho_{N}=\rho_{B}=\frac{2}{3 \sqrt{3} l_{h-B N}^{2}}$ denoting the number of the nitrogen and boron atoms per unit area on h-BN. Here, $l_{C}$ and $l_{h-B N}$ are the equilibrium bond length of graphene and h-BN. Let $h$ denote the equilibrium distance between these two layers. The distance between a carbon atom on graphene and an atom (nitrogen or boron) on h-BN is $r=\sqrt{h^{2}+z^{2}}$, where $z$ is the projected distance between the two atoms on each layer [see Figure 1(a)]. The cohesive energy $\Phi$ is the energy per unit area and is given by

$$
\Phi_{\text {graphene } / h-B N}=\Phi_{C-N}+\Phi_{C-B},
$$

where $\Phi_{C-N}$ and $\Phi_{C-B}$ are the energy due to the interaction between carbon and nitrogen atoms, and carbon and boron atoms, respectively, and they are given by

$$
\begin{aligned}
\Phi_{C-N} & =2 \pi \rho_{C} \rho_{N} \int_{0}^{\infty} V_{C-N}(r) z d z \\
& =2 \pi \rho_{C} \rho_{N} \varepsilon_{C-N} \sigma_{C-N}^{2}\left(\frac{2 \sigma_{C-N}^{10}}{5 h^{10}}-\frac{\sigma_{C-N}^{4}}{h^{4}}\right),
\end{aligned}
$$

and

$$
\begin{aligned}
\Phi_{C-B} & =2 \pi \rho_{C} \rho_{B} \int_{0}^{\infty} V_{C-B}(r) z d z \\
& =2 \pi \rho_{C} \rho_{B} \varepsilon_{C-B} \sigma_{C-B}^{2}\left(\frac{2 \sigma_{C-B}^{10}}{5 h^{10}}-\frac{\sigma_{C-B}^{4}}{h^{4}}\right) .
\end{aligned}
$$

Minimization of the total cohesive energy, $\frac{\partial \Phi_{\text {graphene } / h-B N}}{\partial h}=0$, gives the equilibrium distance $h_{e}$ as

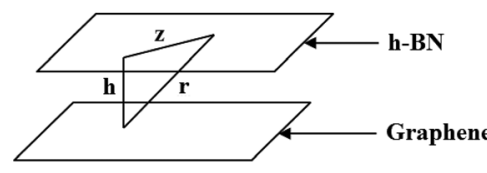

(a)

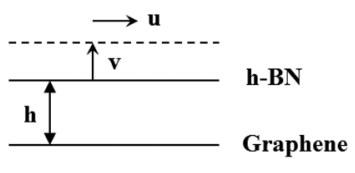

(b)
FIG. 1. (a) Schematic diagram of graphene/monolayer h-BN, (b) the monolayer h-BN is subjected to the opening displacement $v$ and sliding displacement $u$.

$$
h_{e}=\left(\frac{\varepsilon_{C-N} \sigma_{C-N}^{12}+\varepsilon_{C-B} \sigma_{C-B}^{12}}{\varepsilon_{C-N} \sigma_{C-N}^{6}+\varepsilon_{C-B} \sigma_{C-B}^{6}}\right)^{\frac{1}{6}} .
$$

For the opening displacement $v$ and sliding displacement $u$ beyond the equilibrium distance $h_{e}$, as shown in Figure 1(b), the cohesive energy can be similarly obtained as

$$
\begin{aligned}
& \Phi_{\text {graphene } / h-B N}(u, v) \\
& =2 \pi \rho_{C} \rho_{N} \varepsilon_{C-N} \sigma_{C-N}^{2}\left(\frac{2 \sigma_{C-N}^{10}}{5\left(h_{e}+v\right)^{10}}-\frac{\sigma_{C-N}^{4}}{\left(h_{e}+v\right)^{4}}\right) \\
& \quad+2 \pi \rho_{C} \rho_{B} \varepsilon_{C-B} \sigma_{C-B}^{2}\left(\frac{2 \sigma_{C-B}^{10}}{5\left(h_{e}+v\right)^{10}}-\frac{\sigma_{C-B}^{4}}{\left(h_{e}+v\right)^{4}}\right) .
\end{aligned}
$$

This leads to a vanishing shear cohesive stress

$$
\tau_{\text {cohesive }}=\frac{\partial \Phi_{\text {graphene } / h-B N}(u, v)}{\partial u}=0
$$

and a tensile cohesive stress

$$
\begin{aligned}
\sigma_{\text {cohesive }}= & \frac{\partial \Phi_{\text {graphene } / h-B N}(u, v)}{\partial v} \\
= & 8 \pi \rho_{C} \rho_{N} \varepsilon_{C-N} \sigma_{C-N}\left(\frac{\sigma_{C-N}^{5}}{\left(h_{e}+v\right)^{5}}-\frac{\sigma_{C-N}^{11}}{\left(h_{e}+v\right)^{11}}\right) \\
& +8 \pi \rho_{C} \rho_{B} \varepsilon_{C-B} \sigma_{C-B}\left(\frac{\sigma_{C-B}^{5}}{\left(h_{e}+v\right)^{5}}-\frac{\sigma_{C-B}^{11}}{\left(h_{e}+v\right)^{11}}\right) .
\end{aligned}
$$

Given the bond length of graphene prior to deformation, $l_{C}=0.1408 \mathrm{~nm}$ and $\mathrm{h}-\mathrm{BN}, l_{h-B N}=0.1440 \mathrm{~nm},{ }^{45}$ the densities are obtained as $\rho_{C}=38.80 \mathrm{~nm}^{-2}$ and $\rho_{N}=\rho_{B}$ $=18.56 \mathrm{~nm}^{-2}$. The equilibrium distance between graphene and monolayer h-BN is given by Eq. (5) as $h_{G / h B N}^{(e)} \approx 0.3388$ $\mathrm{nm}$. Figure 2 shows the cohesive stress versus the opening displacement for graphene and monolayer h-BN. The critical separation $\delta_{G / h B N} \approx 0.0476 \mathrm{~nm}$ at which the cohesive strength (maximum cohesive stress) is reached as $\sigma_{G / h B N}$ $\approx 2.04 \mathrm{GPa}$. The total cohesive energy (area underneath the $\sigma_{\text {cohesive }}-v$ curve) is $\Phi_{G / h B N} \approx 0.367 \mathrm{~J} / \mathrm{m}^{2}$.

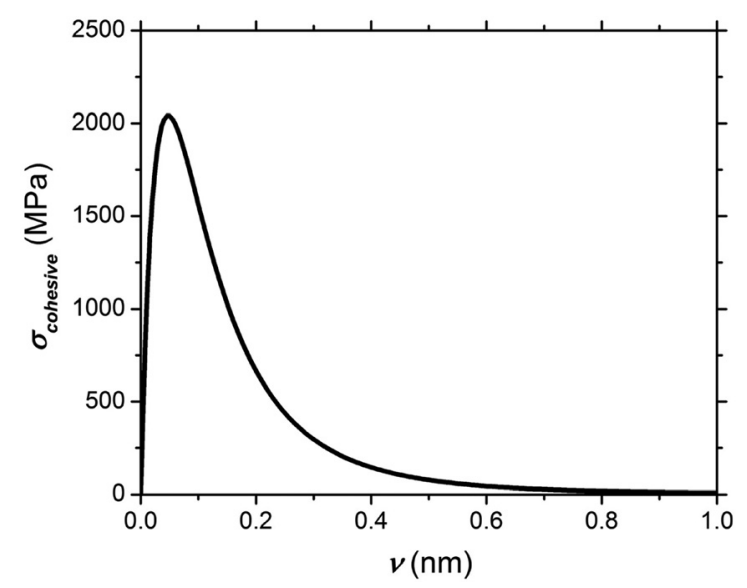

FIG. 2. The cohesive stress $\sigma_{\text {cohesive }}$ versus the opening displacement $v$ for graphene/monolayer h-BN. 


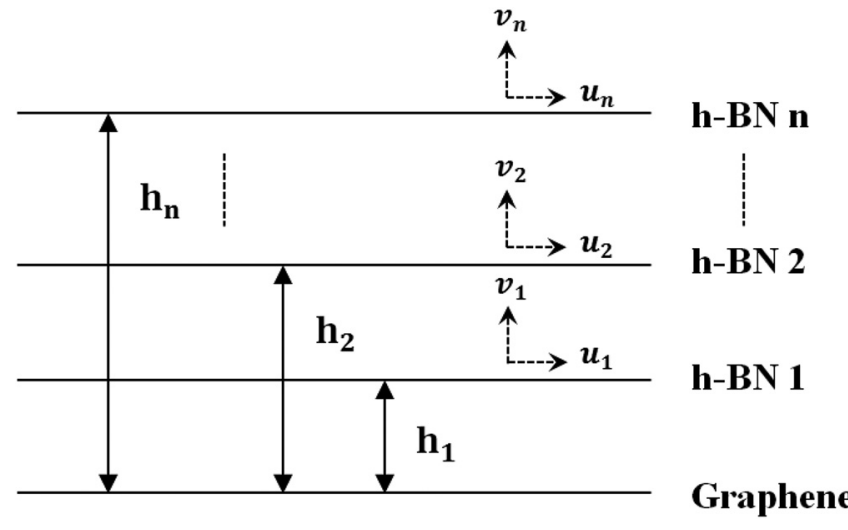

FIG. 3. Schematic diagram of graphene/multi-layer h-BN.

\section{COHESIVE LAW FOR GRAPHENE/MULTI-LAYER h-BN}

The cohesive energy for graphene/multi-layer h-BN consists of the van der Waals interactions among h-BN layers and that between graphene and h-BN layers. Figure 3 shows the schematic diagram of graphene/multi-layer h-BN with $h_{i}$ denoting the equilibrium distance between graphene and the $i$ th layer h-BN, $u_{i}$ and $v_{i}$ denoting the sliding and opening displacements of $i$ th h-BN layer beyond the equilibrium distance. $i=1$ corresponds to the h-BN layer closest to graphene and $i=n$ corresponds to the outmost layer.

Similar to the cohesive energy between graphene and monolayer h-BN, the cohesive energy between the $i$ th and $j$ th h-BN $(i<j)$ is

$$
\begin{aligned}
\Phi_{i-h-B N / j-h-B N} \\
=2 \pi \rho_{N}^{2} \varepsilon_{N-N} \sigma_{N-N}^{2}\left(\frac{2 \sigma_{N-N}^{10}}{5\left(h_{j}-h_{i}\right)^{10}}-\frac{\sigma_{N-N}^{4}}{\left(h_{j}-h_{i}\right)^{4}}\right) \\
+4 \pi \rho_{N} \rho_{B} \varepsilon_{N-B} \sigma_{N-B}^{2}\left(\frac{2 \sigma_{N-B}^{10}}{5\left(h_{j}-h_{i}\right)^{10}}-\frac{\sigma_{N-B}^{4}}{\left(h_{j}-h_{i}\right)^{4}}\right) \\
+2 \pi \rho_{B}{ }^{2} \varepsilon_{B-B} \sigma_{B-B}^{2}\left(\frac{2 \sigma_{B-B}^{10}}{5\left(h_{j}-h_{i}\right)^{10}}-\frac{\sigma_{B-B}^{4}}{\left(h_{j}-h_{i}\right)^{4}}\right)
\end{aligned}
$$

where $\varepsilon_{N-N}=0.006283 \mathrm{eV}$ and $\sigma_{N-N}=0.3365 \mathrm{~nm}$ are the bond energy and equilibrium distance for a pair of nitrogen atoms, $\varepsilon_{N-B}=0.005084 \mathrm{eV}$ and $\sigma_{N-B}=0.3409 \mathrm{~nm}$ for a pair of nitrogen and boron atoms, and $\varepsilon_{B-B}=0.004117 \mathrm{eV}$ and $\sigma_{B-B}=0.3453 \mathrm{~nm}$ for a pair of boron atoms. ${ }^{44}$ For monolayer h-BN and monolayer h-BN, the equilibrium distance can be obtained by minimizing the above energy as $h_{h B N / h B N}^{(e)} \approx 0.3407 \mathrm{~nm}$. The total cohesive energy among all $\mathrm{h}-\mathrm{BN}$ layers is then obtained by

$$
\Phi_{\text {total } h-B N}=\sum_{1 \leq i<j \leq n} \Phi_{i-h-B N / j-h-B N} .
$$

Replacing $h$ in Eq. (2) by $h_{i}$ gives the cohesive energy between the graphene and $i$ th h-BN layers

$$
\begin{aligned}
\Phi_{\text {graphene } / i-h-B N}= & 2 \pi \rho_{C} \rho_{N} \varepsilon_{C-N} \sigma_{C-N}^{2}\left(\frac{2 \sigma_{C-N}^{10}}{5 h_{i}^{10}}-\frac{\sigma_{C-N}^{4}}{h_{i}^{4}}\right) \\
& +2 \pi \rho_{C} \rho_{B} \varepsilon_{C-B} \sigma_{C-B}^{2}\left(\frac{2 \sigma_{C-B}^{10}}{5 h_{i}^{10}}-\frac{\sigma_{C-B}^{4}}{h_{i}^{4}}\right) .
\end{aligned}
$$

The total cohesive energy between the graphene and all $\mathrm{h}-\mathrm{BN}$ layers is then obtained as

$$
\Phi_{\text {graphene/all } h-B N}=\sum_{1 \leq i \leq n} \Phi_{\text {graphene } / i-h-B N} .
$$

The total cohesive energy in graphene/multi-layer h-BN is summation of energy among all h-BN layers in Eq. (10) and energy between graphene and all h-BN layers in Eq. (12), i.e.,

$$
\Phi_{\text {total }}=\Phi_{\text {graphene } / \text { all } h-B N}+\Phi_{\text {total } h-B N}
$$

The equilibrium distances can be determined by minimizing the energy, $\partial \Phi_{\text {total }} / \partial h_{k}=0$, which gives

$$
\begin{aligned}
& 8 \pi \rho_{C} \rho_{N} \varepsilon_{C-N} \sigma_{C-N}\left(\frac{\sigma_{C-N}^{5}}{h_{k}^{5}}-\frac{\sigma_{C-N}^{11}}{h_{k}^{11}}\right)+8 \pi \rho_{C} \rho_{B} \varepsilon_{C-B} \sigma_{C-B}\left(\frac{\sigma_{C-B}^{5}}{h_{k}^{5}}-\frac{\sigma_{C-B}^{11}}{h_{k}^{11}}\right) \\
& +8 \pi \rho_{N}^{2} \varepsilon_{N-N} \sigma_{N-N} \sum_{1 \leq i<k}\left(\frac{\sigma_{N-N}^{5}}{\left(h_{k}-h_{i}\right)^{5}}-\frac{\sigma_{N-N}^{11}}{\left(h_{k}-h_{i}\right)^{11}}\right)-8 \pi \rho_{N}{ }^{2} \varepsilon_{N-N} \sigma_{N-N} \sum_{k<j \leq n}\left(\frac{\sigma_{N-N}^{5}}{\left(h_{j}-h_{k}\right)^{5}}-\frac{\sigma_{N-N}^{11}}{\left(h_{j}-h_{k}\right)^{11}}\right) \\
& +16 \pi \rho_{N} \rho_{B} \varepsilon_{N-B} \sigma_{N-B} \sum_{1 \leq i<k}\left(\frac{\sigma_{N-B}^{5}}{\left(h_{k}-h_{i}\right)^{5}}-\frac{\sigma_{N-B}^{11}}{\left(h_{k}-h_{i}\right)^{11}}\right)+8 \pi \rho_{B}{ }^{2} \varepsilon_{B-B} \sigma_{B-B} \sum_{1 \leq i<k}\left(\frac{\sigma_{B-B}^{5}}{\left(h_{k}-h_{i}\right)^{5}}-\frac{\sigma_{B-B}^{11}}{\left(h_{k}-h_{i}\right)^{11}}\right) \\
& \quad-16 \pi \rho_{N} \rho_{B} \varepsilon_{N-B} \sigma_{N-B} \sum_{k<j \leq n}\left(\frac{\sigma_{N-B}^{5}}{\left(h_{j}-h_{k}\right)^{5}}-\frac{\sigma_{N-B}^{11}}{\left(h_{j}-h_{k}\right)^{11}}\right)-8 \pi \rho_{B}{ }^{2} \varepsilon_{B-B} \sigma_{B-B} \sum_{k<j \leq n}\left(\frac{\sigma_{B-B}^{5}}{\left(h_{j}-h_{k}\right)^{5}}-\frac{\sigma_{B-B}^{11}}{\left(h_{j}-h_{k}\right)^{11}}\right)=0,
\end{aligned}
$$

for $1 \leq k \leq n$, where the terms with $\left(h_{k}-h_{i}\right)$ and the terms with $\left(h_{j}-h_{k}\right)$ disappear for the layer next to the graphene $(k=1)$ and the outmost layer $(k=n)$, respectively. The numerical solution of the above equations gives the equilibrium distance $h_{1}$ between the h-BN and graphene and those between h-BN layers $\left(h_{2}-h_{1}\right),\left(h_{3}-h_{2}\right), \ldots,\left(h_{n}-h_{n-1}\right)$ as 
- $n=1: h_{1}=h_{G / h B N}^{(e)}$;

- $n=2: h_{1}=0.995 h_{G / h B N}^{(e)}, h_{2}-h_{1}=0.996 h_{h B N / h B N}^{(e)}$;

- $n=3: h_{1}=0.994 h_{G / h B N}^{(e)}, h_{2}-h_{1}=0.991 h_{h B N / h B N}^{(e)}$,

$h_{3}-h_{2}=0.994 h_{h B N / h B N}^{(e)}$;

- :

It is observed that the equilibrium distance $h_{1}$ between the h-BN and graphene is very close to $h_{G / h B N}^{(e)}$ and the equilibrium distances between h-BN layers $\left(h_{2}-h_{1}\right), \quad\left(h_{3}-h_{2}\right), \ldots, \quad\left(h_{n}-h_{n-1}\right)$ are very close to $h_{h B N / h B N}^{(e)}$. As $n$ increases, these distances decrease due to the van der Waals interaction from more h-BN layers. This effect becomes negligible once $n$ is larger than 3 , which may suggest that the cohesive law is dominated by the first $3 \mathrm{~h}$-BN layers.

For the opening displacement $v_{i}$ and sliding displacement $u_{i}$ beyond the equilibrium distance $h_{i}(i=1,2,3, \ldots, n)$, as shown in Figure 3, the cohesive energy can be obtained by replacing $h_{i}$ with $\left(h_{i}+v_{i}\right)$ as

$$
\begin{aligned}
\Phi_{\text {total }}(v)= & 2 \pi \rho_{C} \rho_{N} \varepsilon_{C-N} \sigma_{C-N}^{2} \sum_{1 \leq i \leq n}\left(\frac{2 \sigma_{C-N}^{10}}{5\left(h_{i}+v_{i}\right)^{10}}-\frac{\sigma_{C-N}^{4}}{\left(h_{i}+v_{i}\right)^{4}}\right)+2 \pi \rho_{C} \rho_{B} \varepsilon_{C-B} \sigma_{C-B}^{2} \sum_{1 \leq i \leq n}\left(\frac{2 \sigma_{C-B}^{10}}{5\left(h_{i}+v_{i}\right)^{10}}-\frac{\sigma_{C-B}^{4}}{\left(h_{i}+v_{i}\right)^{4}}\right) \\
& +2 \pi \rho_{N}^{2} \varepsilon_{N-N} \sigma_{N-N}^{2} \sum_{1 \leq i<j \leq n}\left(\frac{2 \sigma_{N-N}^{10}}{5\left(h_{j}+v_{j}-h_{i}-v_{i}\right)^{10}}-\frac{\sigma_{N-N}^{4}}{\left(h_{j}+v_{j}-h_{i}-v_{i}\right)^{4}}\right) \\
& +4 \pi \rho_{N} \rho_{B} \varepsilon_{N-B} \sigma_{N-B}^{2} \sum_{1 \leq i<j \leq n}\left(\frac{2 \sigma_{N-B}^{10}}{5\left(h_{j}+v_{j}-h_{i}-v_{i}\right)^{10}}-\frac{\sigma_{N-B}^{4}}{\left(h_{j}+v_{j}-h_{i}-v_{i}\right)^{4}}\right) \\
& +2 \pi \rho_{B}{ }^{2} \varepsilon_{B-B} \sigma_{B-B}^{2} \sum_{1 \leq i<j \leq n}\left(\frac{2 \sigma_{B-B}^{10}}{5\left(h_{j}+v_{j}-h_{i}-v_{i}\right)^{10}}-\frac{\sigma_{B-B}^{4}}{\left(h_{j}+v_{j}-h_{i}-v_{i}\right)^{4}}\right)
\end{aligned}
$$

$v_{i}$ for $i=2,3, \ldots, n$ can be determined in terms of $v_{1}$ by energy minimization $\frac{\partial \Phi_{\text {total }}(v)}{\partial v_{i}}=0$, that is, $v_{i}=v_{i}\left(v_{1}\right)$. The cohesive shear stress, $\frac{\partial \Phi_{\text {total }}}{\partial u_{1}}$, is zero since the total cohesive energy does not depend on the sliding displacement. It should be noted that the vanishing zero shear stress is due to the assumption of infinite large graphene in the in-plane directions, which is valid for large graphene flakes. The cohesive tensile stress is given by

$$
\begin{aligned}
\sigma_{\text {cohesive }}= & \frac{\partial \Phi_{\text {total }}(v)}{\partial v_{1}}=8 \pi \rho_{N}^{2} \varepsilon_{N-N} \sigma_{N-N} \sum_{1<j \leq n}\left(\frac{\sigma_{N-N}^{11}}{\left(h_{j}+v_{j}-h_{1}-v_{1}\right)^{11}}-\frac{\sigma_{N-N}^{5}}{\left(h_{j}+v_{j}-h_{1}-v_{1}\right)^{5}}\right) \\
& +16 \pi \rho_{N} \rho_{B} \varepsilon_{N-B} \sigma_{N-B} \sum_{1<j \leq n}\left(\frac{\sigma_{N-B}^{11}}{\left(h_{j}+v_{j}-h_{1}-v_{1}\right)^{11}}-\frac{\sigma_{N-B}^{5}}{\left(h_{j}+v_{j}-h_{1}-v_{1}\right)^{5}}\right) \\
& +8 \pi \rho_{B}^{2} \varepsilon_{B-B} \sigma_{B-B} \sum_{1<j \leq n}\left(\frac{\sigma_{B-B}^{11}}{\left(h_{j}+v_{j}-h_{1}-v_{1}\right)^{11}}-\frac{\sigma_{-B}^{5}}{\left(h_{j}+v_{j}-h_{1}-v_{1}\right)^{5}}\right) \\
& -8 \pi \rho_{C} \rho_{N} \varepsilon_{C-N} \sigma_{C-N}\left(\frac{\sigma_{C-N}^{11}}{\left(h_{1}+v_{1}\right)^{11}}-\frac{\sigma_{C-N}^{5}}{\left(h_{1}+v_{1}\right)^{5}}\right)-8 \pi \rho_{C} \rho_{B} \varepsilon_{C-B} \sigma_{C-B}\left(\frac{\sigma_{C-B}^{11}}{\left(h_{1}+v_{1}\right)^{11}}-\frac{\sigma_{C-B}^{5}}{\left(h_{1}+v_{1}\right)^{5}}\right) .
\end{aligned}
$$

This is the cohesive law for graphene/multi-layer h-BN due to the van der Waals force.

Figure 4 shows cohesive stress $\sigma_{\text {cohesive }}$ versus the opening displacement $v_{1}$ for graphene/multi-layer h-BN with 1, 2, 3 , and $10 \mathrm{~h}-\mathrm{BN}$ layers. It is noted that the result for $n=10$ is almost equal to that for $n=3$ (error $<0.5 \%$ ), which suggests that the cohesive law is dominated by the first three h-BN layers closest to the graphene. The maximum cohesive stress and total cohesive energy are 2.04 GPa and $0.367 \mathrm{~J} / \mathrm{m}^{2}$ for one h-BN layer, 2.20 GPa and $0.405 \mathrm{~J} / \mathrm{m}^{2}$ for two h-BN layers, and converge to $2.23 \mathrm{GPa}$ and $0.416 \mathrm{~J} / \mathrm{m}^{2}$ for three or more h-BN layers.

The cohesive law in Eq. (16) can be simplified by introducing the following 3 assumptions:

- $v_{i}=v_{1}$ where $i=2,3, \ldots, n$;

- $h_{1}=\tilde{h}_{G / h B N}=\frac{\sigma_{C-N}+\sigma_{C-B}}{2} \quad($ error $<0.7 \%)$;

- $h_{i}-h_{i-1}=\tilde{h}_{h B N / h B N}=\frac{\sigma_{N-N}+\sigma_{N-B}+\sigma_{B-B}}{3}($ error $<1.4 \%)$ where $i=2,3, \ldots, n$.

The cohesive stress then becomes 


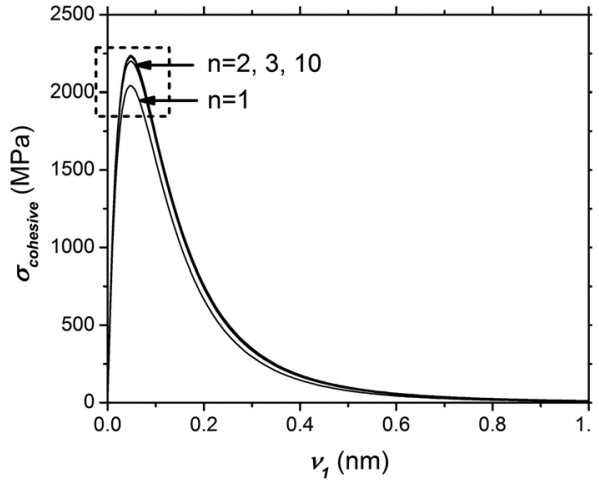

(a)

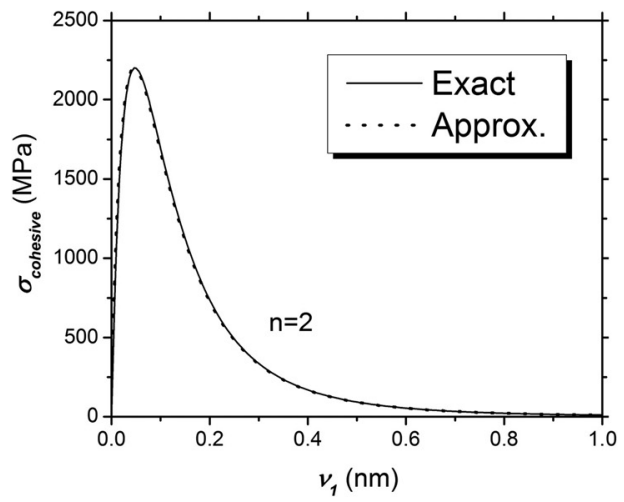

(a)

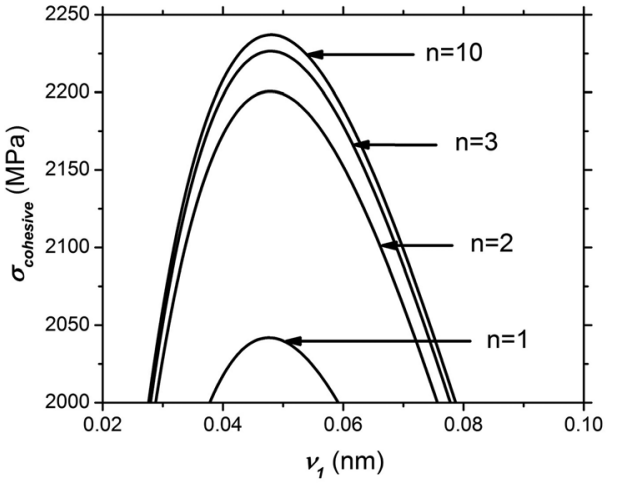

(b)

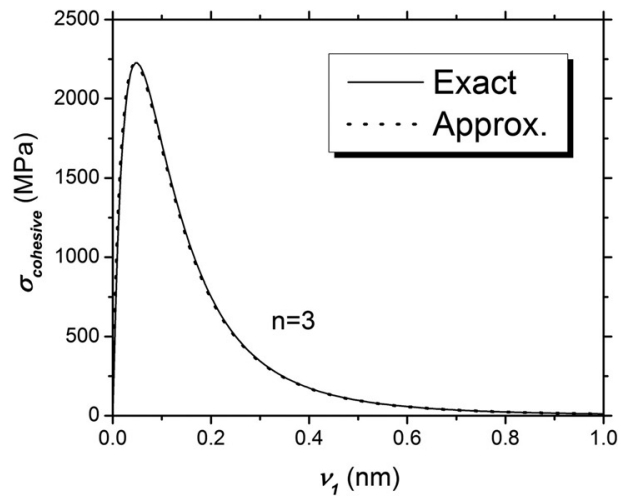

(b)
FIG. 4. (a) The cohesive stress $\sigma_{\text {cohesive }}$ versus the opening displacement $v_{1}$ for graphene/multi-layer h-BN with 1,2 , 3 , and $10 \mathrm{~h}-\mathrm{BN}$ layers. (b) Illustration of the dashed area in (a).
FIG. 5. The comparison of cohesive law between the approximate solution and the exact solution for graphene/multi-layer h-BN with (a) $2 \mathrm{~h}-\mathrm{BN}$ layers and (b) $3 \mathrm{~h}-\mathrm{BN}$ layers.

$$
\begin{gathered}
\sigma_{\text {cohesive }} \approx-8 \pi \rho_{C} \rho_{N} \varepsilon_{C-N} \sigma_{C-N} \sum_{1 \leq i \leq n}\left\{\begin{array}{l}
\frac{\sigma_{C-N}^{11}}{\left[\tilde{h}_{G / h B N}+(i-1) \tilde{h}_{h B N / h B N}+v_{1}\right]^{11}} \\
-\frac{\sigma_{C-N}^{5}}{\left[\tilde{h}_{G / h B N}+(i-1) \tilde{h}_{h B N / h B N}+v_{1}\right]^{5}}
\end{array}\right\} \\
-8 \pi \rho_{C} \rho_{B} \varepsilon_{C-B} \sigma_{C-B} \sum_{1 \leq i \leq n}\left\{\frac{\sigma_{C-B}^{11}}{\left[\tilde{h}_{G / h B N}+(i-1) \tilde{h}_{h B N / h B N}+v_{1}\right]^{11}}\right. \\
-\frac{\sigma_{C-B}^{5}}{\left[\tilde{h}_{G / h B N}+(i-1) \tilde{h}_{h B N / h B N}+v_{1}\right]^{5}}
\end{gathered} .
$$

Figure 5 shows the cohesive law of graphene/multi-layer h-BN for $n=2$ and 3 from the exact solution in Eq. (16) and the approximate solution in Eq. (17). The approximate solution has a good agreement with the accurate solution.

\section{CONCLUSIONS}

We have established the cohesive law for interfaces between graphene and monolayer or multi-layer h-BN based on the van der Waals force. The cohesive law is obtained in terms of the area density of carbon atoms on the graphene and those of boron and nitrogen atoms on the h-BN layer, number of h-BN layer, and the parameters in van der Waals interaction.
It is shown that the cohesive law in the graphene/multi-layer h$\mathrm{BN}$ model is dominated by the first three h-BN layers which are closest to graphene. An approximate solution for the cohesive law is also obtained to give simpler expression. For graphene/h-BN heterostructure with more than three layers of $\mathrm{h}-\mathrm{BN}$, only the summation for $i=1,2$, and 3 are needed since the contribution of further layers can be neglected.

\section{ACKNOWLEDGMENTS}

J.S. acknowledges the supports from NSFC (Grant No. 11372272) and the Thousand Young Talents Program of China. 
${ }^{1}$ K. S. Novoselov, A. K. Geim, S. V. Morozov, D. Jiang, Y. Zhang, S. V. Dubonos, I. V. Grigorieva, and A. A. Firsov, Science 306, 666 (2004).

${ }^{2}$ J. C. Meyer, A. K. Geim, M. I. Katsnelson, K. S. Novoselov, T. J. Booth, and S. Roth, Nature 446, 60 (2007).

${ }^{3}$ M. H. Gass, U. Bangert, A. L. Bleloch, P. Wang, R. R. Nair, and A. K. Geim, Nature Nanotechnol. 3, 676 (2008).

${ }^{4}$ J. C. Meyer, C. Kisielowski, R. Erni, M. D. Rossell, M. F. Crommie, and A. Zettl, Nano Lett. 8, 3582 (2008).

${ }^{5}$ C. Berger, Z. Song, X. Li, X. Wu, N. Brown, C. Naud, D. Mayou, T. Li, J. Hass, A. N. Marchenkov, E. H. Conrad, P. N. First, and W. A. De Heer, Science 312, 1191 (2006).

${ }^{6}$ M. Sprinkle, P. Soukiassian, W. A. De Heer, C. Berger, and E. H. Conrad, Phys. Status Solidi (RRL) 3, A91 (2009).

${ }^{7}$ A. Mattausch and O. Pankratov, Phys. Rev. Lett. 99, 076802 (2007).

${ }^{8}$ K. A. Ritter and J. W. Lyding, Nature Mater. 8, 235 (2009).

${ }^{9}$ K. A. Ritter and J. W. Lyding, Nanotechnology 19, 015704 (2008).

${ }^{10}$ J. D. Caldwell, T. J. Anderson, J. C. Culbertson, G. G. Jernigan, K. D. Hobart, F. J. Kub, M. J. Tadjer, J. L. Tedesco, J. K. Hite, M. A. Mastro, R. L. Myers-Ward, C. R. Eddy, Jr., P. M. Campbell, and D. K. Gaskill, ACS Nano 4, 1108 (2010).

${ }^{11}$ X. Du, I. Skachko, A. Barker, and E. Y. Andrei, Nature Nanotechnol. 3, 491 (2008).

${ }^{12}$ E. V. Castro, H. Ochoa, M. I. Katsnelson, R. V. Gorbachev, D. C. Elias, K. S. Novoselov, A. K. Geim, and F. Guinea, Phys. Rev. Lett. 105, 266601 (2010).

${ }^{13}$ T. Ando, J. Phys. Soc. Jpn. 75, 074716 (2006).

${ }^{14}$ K. Nomura and A. H. MacDonald, Phys. Rev. Lett. 98, 076602 (2007).

${ }^{15}$ E. H. Hwang, S. Adam, and S. D. Sarma, Phys. Rev. Lett. 98, 186806 (2007).

${ }^{16}$ Y. B. Zhang, V. W. Brar, C. Girit, A. Zettl, and M. F. Crommie, Nat. Phys. 5, 722-726 (2009).

${ }^{17}$ M. I. Katsnelson and A. K. Geim, Philos. Trans. R. Soc., A 366, 195 (2008).

${ }^{18}$ M. Ishigami, J. H. Chen, W. G. Cullen, M. S. Fuhrer, and E. D. Williams, Nano Lett. 7, 1643 (2007).

${ }^{19}$ J. H. Chen, C. Jang, S. Xiao, M. Ishigami, and M. S. Fuhrer, Nature Nanotechnol. 3, 206 (2008).

${ }^{20}$ S. Fratini and F. Guinea, Phys. Rev. B 77, 195415 (2008).

${ }^{21}$ K. Watanabe, T. Taniguchi, and H. Kanda, Nature Mater. 3, 404-409 (2004).

${ }^{22}$ S. Cho and M. S. Fuhrer, Phys. Rev. B 77, 081402 (2008).

${ }^{23}$ S. Majety, J. Li, W. P. Zhao, B. Huang, S. H. Wei, J. Y. Lin, and H. X. Jiang, Appl. Phys. Lett. 102, 213505 (2013).
${ }^{24}$ C. R. Dean, A. F. Young, I. Meric, C. Lee, L. Wang, S. Sorgenfrei, K. Watanabe, T. Taniguchi, P. Kim, K. L. Shepard, and J. Hone, Nat. Nanotechnol. 5, 722-726 (2010).

${ }^{25}$ Y. Ding, Y. L. Wang, and J. Ni, Appl. Phys. Lett. 95, 123105 (2009).

${ }^{26}$ J. Sławińska, I. Zasada, P. Kosiński, and Z. Klusek, Phys. Rev. B 82, 085431 (2010).

${ }^{27}$ M. Bokdam, P. A. Khomyakov, G. Brocks, Z. C. Zhong, and P. J. Kelly, Nano Lett. 11, 4631-4635 (2011).

${ }^{28}$ S. Jungthawan, S. Limpijumnong, and J. L. Kou, Phys. Rev. B 84, 235424 (2011).

${ }^{29}$ G. Seol and J. Guo, Appl. Phys. Lett. 98, 143107 (2011).

${ }^{30}$ T. P. Kaloni, Y. C. Cheng, and U. Schwingenschlögl, J. Mater. Chem. 22, 919-922 (2012).

${ }^{31}$ R. Balu, X. L. Zhong, R. Pandey, and S. P. Karna, Appl. Phys. Lett. 100, 052104 (2012).

${ }^{32}$ O. V. Yazyev and A. Pasquarello, Phys. Rev. B 80, 035408 (2009).

${ }^{33}$ X. L. Ding, H. Sun, X. M. Xie, H. C. Ren, F. Q. Huang, and M. H. Jiang, Phys. Rev. B 84, 174417 (2011).

${ }^{34}$ J. W. Jiang, J. S. Wang, and B. S. Wang, Appl. Phys. Lett. 99, 043109 (2011).

${ }^{35}$ M. S. Bresnehan, M. J. Hollander, M. Wetherington, M. LaBella, K. A. Trumbull, R. Cavalero, D. W. Snyder, and J. A. Robinson, ACS Nano 6, 5234-5241 (2012).

${ }^{36}$ N. Jain, T. Bansal, C. A. Durcan, Y. Xu, and B. Yu, Carbon 54, 396-402 (2013).

${ }^{37}$ L. Gong, I. A. Kinloch, R. J. Young, I. Riaz, R. Jalil, and K. S. Novoselov, Adv. Mater. 22, 2694-2697 (2010).

${ }^{38}$ G. Tsoukleri, J. Parthenios, K. Papagelis, R. Jalil, A. C. Ferrari, A. K. Geim, K. S. Novoselov, and C. Galiotis, Small 5, 2397-2402 (2009).

${ }^{39}$ T. Jiang, R. Huang, and Y. Zhu, Adv. Funct. Mater. 24, 396-402 (2014).

${ }^{40}$ L. Y. Jiang, Y. Huang, H. Jiang, G. Ravichandran, H. Gao, K. C. Hwang, and B. Liu, J. Mech. Phys. Solids 54, 2436-2452 (2006).

${ }^{41}$ W. B. Lu, J. Wu, L. Y. Jiang, Y. Huang, K. C. Hwang, and B. Liu, Philos. Mag. 87, 2221-2232 (2007).

${ }^{42}$ W. B. Lu, J. Wu, J. Song, K. C. Hwang, L. Y. Jiang, and Y. Huang, Comput. Methods Appl. Mech. Eng. 197, 3261-3267 (2008).

${ }^{43}$ B. Sachs, T. O. Wehling, M. I. Katsnelson, and A. I. Lichtenstein, Phys. Rev. B 84, 195414 (2011).

${ }^{44}$ N. Thamwattana and J. M. Hill, Physica B 404, 3906-3910 (2009).

${ }^{45}$ B. Altintas, C. Parlak, C. Bozkurt, and R. Eryiğit, Eur. Phys. J. B 79, 301-312 (2011) 